\title{
Comparative analysis of the surface exposed proteome of two canine osteosarcoma cell lines and normal canine osteoblasts
}

Milan Milovancev ${ }^{1 *}$, lan Hilgart-Martiszus ${ }^{1}$, Michael J McNamara ${ }^{2}$, Cheri P Goodall ${ }^{1}$, Bernard Seguin ${ }^{1,4}$, Shay Bracha ${ }^{1}$ and Samanthi I Wickramasekara ${ }^{3}$

\begin{abstract}
Background: Osteosarcoma (OSA) is the most common primary bone tumor of dogs and carries a poor prognosis despite aggressive treatment. An improved understanding of the biology of OSA is critically needed to allow for development of novel diagnostic, prognostic, and therapeutic tools. The surface-exposed proteome (SEP) of a cancerous cell includes a multifarious array of proteins critical to cellular processes such as proliferation, migration, adhesion, and inter-cellular communication. The specific aim of this study was to define a SEP profile of two validated canine OSA cell lines and a normal canine osteoblast cell line utilizing a biotinylation/streptavidin system to selectively label, purify, and identify surface-exposed proteins by mass spectrometry (MS) analysis. Additionally, we sought to validate a subset of our MS-based observations via quantitative real-time PCR, Western blot and semiquantitative immunocytochemistry. Our hypothesis was that MS would detect differences in the SEP composition between the OSA and the normal osteoblast cells.
\end{abstract}

Results: Shotgun MS identified 133 putative surface proteins when output from all samples were combined, with good consistency between biological replicates. Eleven of the MS-detected proteins underwent analysis of gene expression by PCR, all of which were actively transcribed, but varied in expression level. Western blot of whole cell lysates from all three cell lines was effective for Thrombospondin-1, CYR61 and CD44, and indicated that all three proteins were present in each cell line. Semi-quantitative immunofluorescence indicated that CD44 was expressed at much higher levels on the surface of the OSA than the normal osteoblast cell lines.

Conclusions: The results of the present study identified numerous differences, and similarities, in the SEP of canine OSA cell lines and normal canine osteoblasts. The PCR, Western blot, and immunocytochemistry results, for the subset of proteins evaluated, were generally supportive of the mass spectrometry data. These methods may be applied to other cell lines, or other biological materials, to highlight unique and previously unrecognized differences between samples. While this study yielded data that may prove useful for OSA researchers and clinicians, further refinements of the described techniques are expected to yield greater accuracy and produce a more thorough SEP analysis.

Keywords: Dog, Proteomics, Osteosarcoma, Mass spectrometry, Biotinylation

\footnotetext{
* Correspondence: milan.milovancev@oregonstate.edu

${ }^{1}$ Department of Clinical Sciences, College of Veterinary Medicine, Oregon

State University, Corvallis, OR 97331, USA

Full list of author information is available at the end of the article
} 


\section{Background}

Osteosarcoma (OSA) is the most common primary bone tumor of dogs with $>8000$ cases diagnosed annually in the United States of America [1]. Despite aggressive treatment incorporating surgery, radiation therapy, and/ or chemotherapy, survival times remain poor. Over $90 \%$ of canine patients undergoing standard-of-care treatment, without clinically detectable metastasis at time of diagnosis, will ultimately succumb to metastatic disease $[1,2]$. Overall reported median survival times are 235366 days, and 1- and 2-year survival rates range from $33-65 \%$ and $16-28 \%$, respectively [1-4]. The development of effective tools to diagnose and treat OSA is essential for mitigating the negative impact of this disease on both canine patients and their owners.

The extracellular surface of a cancerous cell includes a multifarious array of proteins that are critical to processes such as cell proliferation, migration, adhesion, and intercellular communication. Collectively, this group of proteins can be referred to as the surface-exposed proteome (SEP). Abnormalities in the SEP are central to the biologic behavior of cancer [5]. Recognized abnormalities in cancerous cells include changes in the expression level of particular proteins, the presence of unusual protein isoforms, and alterations in post-translational modification patterns (e.g. phosphorylation or glycosylation) $[5,6]$.

Despite OSA's considerable clinical impact, a comparative analysis of the SEP between canine osteosarcoma and normal osteoblast cells has not been described in the veterinary literature. Isolated reports of specific abnormalities in canine OSA are limited to focused evaluations of specific proteins, often prompted by discoveries in other neoplasms or species. Examples of such proteins include: survivin [7], cathepsin K [8], Met [9-11], EGFR [9], Ron [9], p53 [12,13], HER-2 [14], HGF [10,11], IGF-1 and IGF1R [15], MMP-2 and MMP-9 [16,17], and ezrin [18]. A more global analysis of the SEP differences between OSA and normal osteoblast cells holds the potential of identifying unique and previously unrecognized features of OSA that can be exploited in treatment, diagnostic, and/or prognostic capacities [5,6,19-23].

While there are many different methods available for enriching plasma membrane proteins, labeling surface proteins with biotin is a popular approach because of the availability of membrane-impermeable biotinylation reagents and the strong association between biotin and streptavidin, which is used for affinity purification of biotin-labeled molecules [24]. These two attributes make it feasible to selectively label surface proteins, and then use denaturing conditions to extract total protein from labeled cells and affinity purify the biotinylated fraction. The purified surface proteins can then be enzymatically digested with trypsin and the resulting peptides identified by mass spectrometry (MS). MS-based proteomics has been applied to numerous cancer biomarker discovery projects and enables protein-level investigations reflecting many of the biological processes relevant to cancer growth and invasion [20,21,25,26].

The specific aim of our study was to define a profile of proteins that compose the SEP of two validated canine OSA cell lines and a normal canine osteoblast cell line utilizing the aforementioned biotinylation/streptavidin system to selectively label, purify, and identify surfaceexposed proteins by LC-MS/MS analysis. Additionally, we sought to validate a subset of our MS-based observations via quantitative real-time PCR (qRT-PCR) and antibody-based techniques. Our hypothesis was that MS would detect differences in the SEP composition between the OSA and the cultured normal osteoblast cells.

\section{Results}

\section{Identification of surface-exposed proteins}

The biotin-labeled surface proteins from each cell line were extracted and visualized by SDS-PAGE and Western blot (Figure 1). The gross comparison of biotinylation patterns indicated that the complement of surface-exposed proteins from the POS and HMPOS cell lines appeared similar, while the surface proteome of the $\mathrm{CnOb}$ cell line appeared to be markedly different. Following the affinity purification and proteolysis of the biotinylated proteins, analysis of the peptides by shotgun MS identified a total of 133 putative surface proteins when outputs from all samples and replicates were combined (Additional file 1: Table S1). Protein identifications were largely consistent between biological replicates (Additional file 2: Table S2). Based on their potential relevance to cancer biology, a subset of the identified proteins was selected for inclusion in Table 1, with their relative spectral counts expressed as a percentage of total spectra. Several proteins that are known to be associated with the cell surface and/or interact with the extracellular matrix were detected in all three cell lines, such as Fibronectin, Vitronectin, CYR61 and Annexin A2 [27-30]. Several additional surface proteins were found to be detected in abundance in one cell line, but poorly detected or absent in the others. For example, peptides originating from Thrombospondin-1 were observed in abundance from $\mathrm{CnOb}$ samples, but were not observed in any of the replicates from the POS or HMPOS cell lines. Similarly, both Chondroitin Sulfate Proteoglycan 4 (CSPG4) and CD109 were detected from POS samples, but were not detected in the other samples.

\section{qRT-PCR}

Eleven of the proteins that were detected by MS were selected for secondary analysis of gene expression by qRTPCR, the results of which are summarized in Figure 2. These eleven proteins were selected on the basis of their relevance to cancer biology and the availability of 


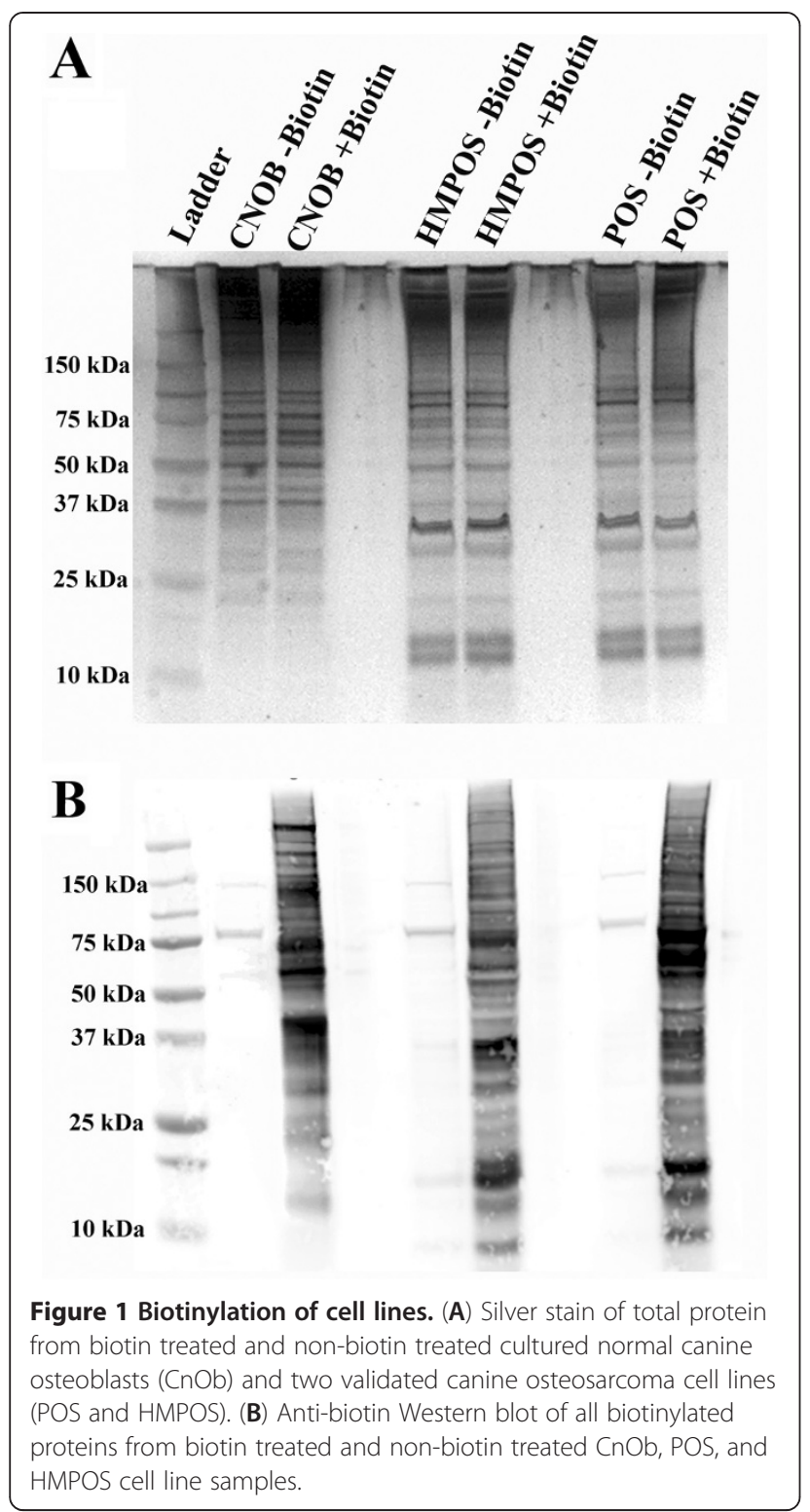

antibodies that were potentially compatible with canine targets. Active transcription of all of the selected genes was detected by qRT-PCR, indicating that all of these genes are indeed expressed in the cell lines used in this study. Expression was normalized to GAPDH and the biological replicates were generally in close agreement between samples, with few minor variations (Additional file 3: Table S3).

\section{Western blot}

Following validation and quantification of gene expression by qRT-PCR, five proteins were selected for further validation by Western blot: Thrombospondin-1, CYR61, CD44, Notch2 and Plexin B2. Due to the dearth of validated, canine-specific antibodies, these targets were selected based on the availability of antibodies that were likely to be cross-reactive with canine proteins. The antibodies against Notch2 and Plexin B2 were found to have poor target specificity towards the canine proteins in our samples and were excluded from the final figure. Western blots of whole cell lysates from all three cell lines was effective for Thrombospondin-1, CYR61 and CD44, and indicated that all three proteins were present in each cell line, albeit with differential abundance (Figure 3).

\section{Immunocytochemistry}

To assess surface-localization of the selected proteins, an on-cell Western blot approach was used to visualize and quantify the proteins on the surface of live cells. Of the five experimental antibodies tested in this study, only the anti-CD44 antibody was validated for either immunocytochemistry or flow cytometry applications, and only with murine cells. Not surprisingly, only the antiCD44 antibody was observed to bind, at detectable levels, to the cell surface of the cell lines used in this study. The fluorescent signal from anti-CD44 was quantified on a fluorescent scanner and normalized to the approximate number of cells, as measured by the signal intensity from the nuclear stain Hoecsht 33342. This semi-quantitative ICC analysis indicated that all three cell lines expressed CD44 on their surface, although it was much less abundant on the surface of $\mathrm{CnOb}$ cells compared to POS or HMPOS cells (Figure 4). The complete ICC results for all of the tested antibodies are summarized in Additional file 4.

\section{Discussion}

The present study successfully employed a method of biotinylation/streptavidin enrichment with MS-based peptide sequencing to selectively label, purify, and identify surface exposed proteins from two validated canine OSA cell lines and a commercially available normal osteoblast line. A total of 133 putative surface-exposed proteins were identified when outcome from all cell lines was combined. Significant differences in SEP composition, and many similarities, were observed between the canine OSA cell lines (POS, HMPOS) and the normal canine osteoblast cell line $(\mathrm{CnOb})$. Based on these observations, we conclude that MS can effectively detect SEP differences between cultured canine OSA and normal osteoblast cell lines.

When evaluating the list of 133 proteins identified via these methods, it is important to bear in mind that confident quantification of protein levels is not possible with this methodology because relative quantities can become skewed during the enrichment process, depending on the protein. Furthermore, this analysis invariably represents only a fraction of the total SEP; as a 
Table 1 Subset of mass spectrometry-identified surface proteins of cell lines

\begin{tabular}{|c|c|c|c|c|c|}
\hline Protein name & Gene name & NCBI Ref Sequence & CnOb & POS & HMPOS \\
\hline Fibronectin & FN1 & XP_536059 & 0.55 & 0.16 & 0.14 \\
\hline Annexin 2 & ANXA2 & NP_001002961 & 0.15 & 0.33 & 0.43 \\
\hline Protein CYR61 & CYR61 & XP_537091 & 0.1 & 0.01 & 0.48 \\
\hline Vitronectin & VTN & XP_854040 & 0.1 & 0.11 & 0.13 \\
\hline Serpin Peptidase Inhibitor & SERPINH1 & NP_001159360 & 0.17 & 0.37 & 0.24 \\
\hline Thrombospondin-1 & THBS1 & XP_544610 & 1.1 & 0 & 0 \\
\hline Chondroitin sulfate proteoglycan 4 & CSPG4 & XP_544783 & 0 & 2.0 & 0 \\
\hline CD109 antigen isoform 3 & CD109 & XP_532205 & 0 & 0.21 & 0 \\
\hline Neuropilin-1 isoform 2 & NRP1 & XP_535142 & 0 & 0.12 & 0 \\
\hline Glypican-4 & GPC4 & XP_549265 & 0 & 0.11 & 0 \\
\hline 4 F2 cell-surface antigen heavy chain & SLC3A2 & XP_540898 & 0 & 0.11 & 0 \\
\hline Cysteine-rich secretory protein LCCL domain-containing 2 & CRISPLD2 & XP_546797 & 0 & 0 & 0.06 \\
\hline Integrin Beta-1 & ITGB1 & XP_535143 & 0.07 & 0.08 & 0 \\
\hline Plexin B2 & PLXNB2 & XP_531689 & 0 & 0.77 & 0.11 \\
\hline CD44 antigen precursor & CD44 & NP_001183951 & 0 & 0.15 & 0.07 \\
\hline Ephrin type-A receptor 2 isoform 1 & $\mathrm{EPHA} 2$ & XP_544546 & 0 & 0.35 & 0.06 \\
\hline Inactive tyrosine-protein kinase 7 isoform A & PTK7 & XP_538929 & 0 & 0.42 & 0.02 \\
\hline C-type mannose receptor 2 & MRC2 & XP_003435244 & 0 & 0.22 & 0.01 \\
\hline Delta-sarcoglycan & SGCD & XP_854897 & 0 & 0.16 & 0.01 \\
\hline Notch homolog protein 2 isoform 1 & $\mathrm{NOTCH} 2$ & XP_540266 & 0 & 0.08 & 0.02 \\
\hline
\end{tabular}

A subset of the surface-exposed proteins identified by mass spectrometry in cultured normal canine osteoblasts ( $\mathrm{CnOb}$ ) and two validated canine osteosarcoma cell lines (POS and HMPOS), with their relative spectral counts expressed as a percentage of total spectra.

comprehensive profile would require the use of complementary sample processing methods, standards, and controls. Despite these limitations, the primary benefit of MS-based proteomic techniques, such as the one described in this report, is the ability to identify numerous previously unrecognized SEP differences between samples in a high-throughput fashion. This latitudinous analysis of the SEP can highlight novel targets for future diagnostic, prognostic and therapeutic methods [5,6,19].

MS-based proteomics is a relatively new tool in canine OSA research, and due to the limited information on this specific disease, we sought to validate a subset of our MS observations via secondary methods. These included qRTPCR, Western blot, and immunocytochemistry. The

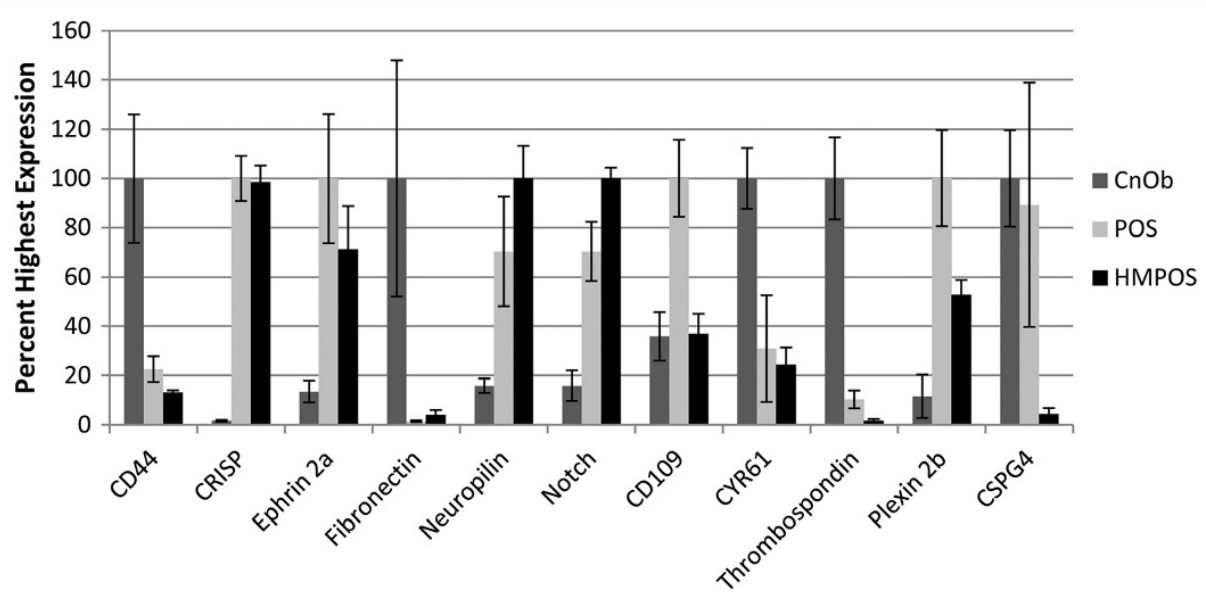

Figure 2 Quantitative real-time PCR of cell lines. Quantitative real-time PCR indicating relative expression of selected genes corresponding to surface proteins detected by mass spectrometry of cultured normal canine osteoblasts ( $\mathrm{CnOb}$ ) and two validated canine osteosarcoma cell lines (POS and HMPOS). 


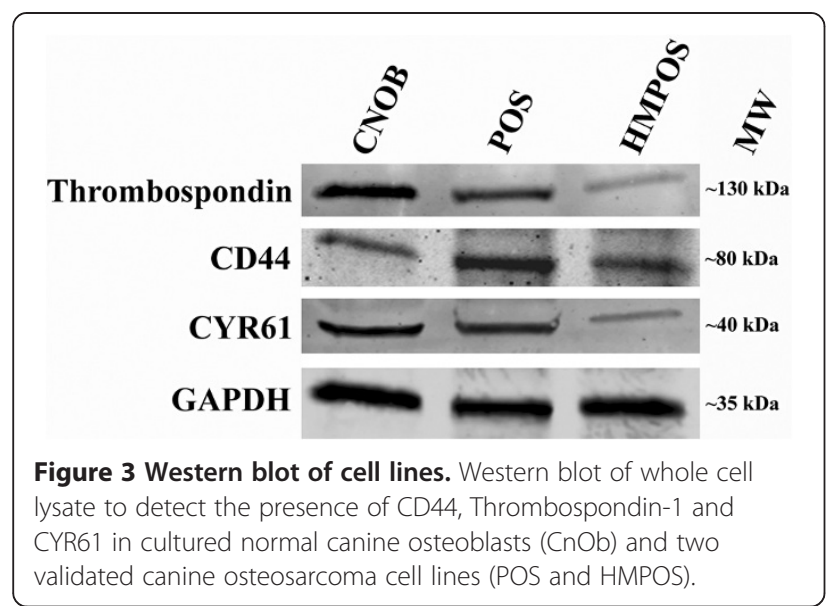

results from these secondary tests were broadly confirmatory, but they occasionally contradictory, and highlighted some important limitations of the MS data sets. These results are summarized for a selected group of proteins in Table 2. For example, a negative result for an antigen in the MS data was not necessarily indicative that the target was absent, which was illustrated by our confirmation of
CD44. Overall, the limited scope of this study means that the resulting data is inherently incomplete. However, these initial results also indicate that further improvements in sample processing methods, mass spectrometry technologies, and canine-specific antibodies will improve the feasibility and utility of this type of analysis.

Our initial supportive experiment was qRT-PCR analysis of 11 of the 133 MS-identified surface-proteins. These 11 proteins were selected based on their known roles in cancer biology and apparent differences in abundance, as measured by MS spectral counts. Our analysis revealed that transcripts for all 11 genes were detected in each of the 3 cell lines, indicating that each of these genes were indeed expressed, and that each protein was potentially produced by each cell line. Protein quantification using spectral counting (label free approach) is considered to be semi-quantitative and the accuracy is based on the length of the protein, reproducibility of the sample preparation techniques, and LC separation [31]. In this study, because affinity enrichment was incorporated into the sample preparation, the relative abundance of the proteins determined by spectral counting can be misleading. Bearing this limitation in mind, the expression levels of several of

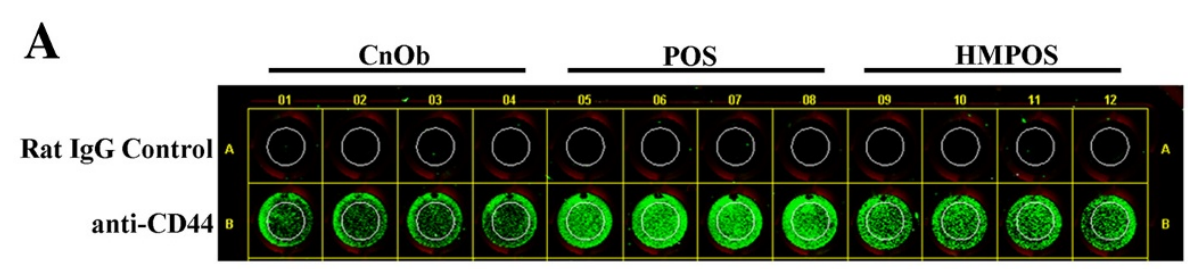

B

anti-CD44 Staining (Normalized MFI)

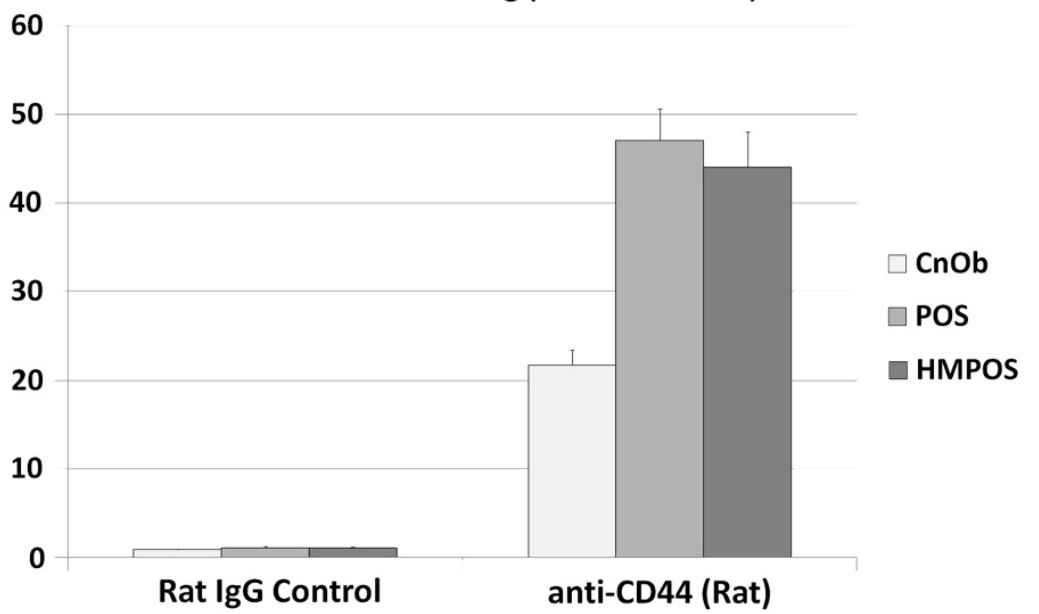

Figure 4 anti-CD44 immunocytochemistry on live cell lines. A. On-cell Western blot image showing binding of anti-CD44 antibody to the surface of live CnOb (normal canine osteoblast cell line), POS and HMPOS (canine osteosarcoma cell lines). B. Semi-quantitative analysis of CD44 density on cell surface indicating increased surface expression in both osteosarcoma cell lines (POS and HMPOS), relative to normal osteoblasts (CnOb). Quantification measures mean fluorescent intensity (MFI) of anti-CD44 staining, normalized to the approximate number of cells (quantified by nuclear stain). 
Table 2 Summary of observations for selected surface proteins

\begin{tabular}{|c|c|c|c|c|}
\hline Protein name & Mass spec & qRT-PCR & Western blot & ICC \\
\hline \multicolumn{5}{|l|}{ Thrombospondin-1 } \\
\hline $\mathrm{CnOb}$ & D & High & High & N/A \\
\hline POS & ND & Low & Mid & N/A \\
\hline HMPOS & ND & Low & Low & N/A \\
\hline \multicolumn{5}{|l|}{ CD44 } \\
\hline $\mathrm{CnOb}$ & ND & High & Low & Low \\
\hline POS & D & Low & High & High \\
\hline HMPOS & $\mathrm{D}$ & Low & Mid & High \\
\hline \multicolumn{5}{|l|}{ CYR61 } \\
\hline $\mathrm{CnOb}$ & D & High & High & N/A \\
\hline POS & D & Low & High & $\mathrm{N} / \mathrm{A}$ \\
\hline HMPOS & D & Low & Low & N/A \\
\hline \multicolumn{5}{|l|}{ Plexin B2 } \\
\hline $\mathrm{CnOb}$ & ND & Low & N/A & $\mathrm{N} / \mathrm{A}$ \\
\hline POS & D & High & N/A & N/A \\
\hline HMPOS & D & Mid & N/A & N/A \\
\hline \multicolumn{5}{|l|}{ Notch-2 } \\
\hline $\mathrm{CnOb}$ & ND & Low & N/A & $\mathrm{N} / \mathrm{A}$ \\
\hline POS & $\mathrm{D}$ & Mid & N/A & N/A \\
\hline HMPOS & $D$ & High & N/A & N/A \\
\hline
\end{tabular}

A summary of observations for a subset of the surface-exposed proteins identified by mass spectrometry in cultured normal canine osteoblasts $(\mathrm{CnOb})$ and two validated canine osteosarcoma cell lines (POS and HMPOS) and the results of their evaluation via secondary techniques (qRT-PCR, Western blot, and immunocytochemistry [ICC]).

D Detected.

ND Not detected.

High, Mid, Low = relative expression levels observed.

the genes matched their apparent abundance at the cell surface, as measured by MS. Most notable among these was Thrombospondin-1, which was detected in unique abundance from $\mathrm{CnOb}$ samples, and whose transcript was also expressed at much higher levels in $\mathrm{CnOb}$ cells than in POS or HMPOS cells. At the same time, the apparent protein surface abundance did not closely match gene expression data for several of the targets. These differences may arise from differences in post-transcriptional regulation and/or protein trafficking to the cell surface, or they may be artifacts of the experimental method. While qRT-PCR demonstrates which genes are expressed and is an indicator of which proteins are likely to be more or less abundant, qRT-PCR is not a direct indicator of the abundance and localization of the corresponding proteins.

Western blot analysis demonstrated that the proteins Thrombospondin-1, CYR61 and CD44 were present at varying levels in all 3 cell lines. These results are compatible with the qRT-PCR results, indicating that these genes are both expressed and translated into protein in the cell lines studied. Again, the results were most consistent for Thrombospondin-1 protein, which was observed by Western Blot to be most abundant in the $\mathrm{CnOb}$ sample. Our MS analysis did not detect thombospondin-1 in the OSA cell lines, although the qRT-PCR and Western Blot results do indicate that this gene is transcribed and translated in the OSA cell lines, albeit at much lower levels than in CnOb. Additionally, CD44 was detected by qRT-PCR and Western blot in all three cell lines, but was not observed by MS in the $\mathrm{CnOb}$ cell line. The Western blot analysis represents all the proteins in the cells (whole cell lysate) whereas our MS-based SEP analysis is designed to specifically target the cell surface proteins. Therefore, the observed differences between Western blot results and MS findings may be due to changes in the protein localization with the cells, or they may represent limitations of our methods. Importantly, none of the proteins identified by MS were found to be absent in any cell line by Western blot (i.e. MS did not spuriously report presence of the three proteins, as verified by Western blot).

Immunocytochemistry represents the most direct method for confirming the surface localization of the proteins in question. However, the lack of validated antibodies for use with canine cells limits the population of proteins that could be confirmed using this technique. While all five of our antibodies were tested for potential reactivity, only one ( $\alpha \mathrm{CD} 44)$ was found to bind to the surface of any of the cell lines, at a detectable level. This was result was not surprising because only the $\alpha \mathrm{CD} 44$ was validated for flow cytometry applications. We observed positive CD44 staining for all of the cell lines, although the signal was much greater in the OSA lines compared to the $\mathrm{CnOb}$ cells. These results are largely consistent with our MS-based SEP analysis and are compatible with our Western blot observations. A previous study, using methods other than MS, has also demonstrated presence of CD44 on the cell surface of cultured canine OSA cells [32]. Furthermore, the CD44 immunocytochemistry findings suggest gene expression levels (assessed by qRT-PCR) and presence of CD44 in the whole cell lysate (assessed by Western blot) may not be indicative of concentration of the protein in question at the cellular surface-an observation that supports the selectivity of the biotin/streptavidin surface-protein enrichment technique employed in the present study.

Although the 133 proteins identified in this study represent a significant contribution to the study of canine OSA biology, they do not represent the entire SEP of these cells. A more comprehensive survey of the SEP composition could be achieved with the addition of complementary sample processing methods. For example, our method used amine-reactive biotinylation followed by Trypsin digestion of the recovered proteins to generate peptides for analysis. A more thorough SEP 
profile might incorporate an alternative biotinylation reagent (e.g. biotin hydrazide) [33] and/or protease (e.g. Glu-C) [34,35]. The addition of these methods would generate complementary data sets, ultimately increasing the depth, breadth, and accuracy of the analysis. Also, simply increasing the physical amount of sample analyzed for each cell line would be expected to yield more peptides for detection via MS, which would in turn increase the detection rate for proteins that exist in low quantities on the cell surface.

\section{Conclusions}

In conclusion, the results of the present study identified numerous similarities and differences in the SEP between cultured canine OSA cell lines and cultured normal canine osteoblasts. The subset of these findings evaluated via secondary techniques including qRT-PCR, Western blot, and immunocytochemistry were found to be generally supportive of the mass spectrometry data, although the inconsistencies also demonstrate the limitations of this analysis (Table 2). These methods may be applied to other cell lines, or other biological materials, to highlight unique and previously unrecognized and unexpected differences between samples. While this study yielded data that may prove useful for OSA researchers and clinicians, further refinements of the described techniques are expected to yield greater accuracy and more comprehensive data sets.

\section{Methods}

\section{Identification of surface-exposed proteins}

The canine osteosarcoma cell lines POS and HMPOS [36] were cultured in RPMI 1640 medium supplemented with $10 \%$ fetal bovine serum and the normal canine osteoblast cell line, $\mathrm{CnOb}$ (Cell Applications, San Diego, CA), was cultured in canine osteoblast medium (Cell Applications, San Diego, CA). All cells were maintained in a humidified incubator with $5 \% \mathrm{CO}_{2}$ at $37^{\circ} \mathrm{C}$.

For all biotinylation experiments, cells were grown to $\sim 80 \%$ confluency in T75 tissue culture flasks (Corning Inc, Corning, NY). Prior to biotinylation, cells were washed 5 times for 5 min with $10 \mathrm{~mL}$ Hanks Buffered Salt Solution (HBSS). The membrane-impermeable biotinylation reagent Sulfo-NHS-LC-Biotin (Pierce, Rockford, IL) was used to covalently label the surface-exposed proteins from live cultures of $\mathrm{CnOb}$, POS and HMPOS cells: $5 \mathrm{mg}$ of Sulfo-NHS-LC-Biotin (Pierce, Rockford, IL) was dissolved in $10 \mathrm{~mL}$ of HBSS and added to each flask. For negative controls (no biotinylation), $10 \mathrm{ml}$ of plain HBSS was added to each flask. The biotinylation reaction was carried out in the tissue culture vessel, with gentle shaking, for 30 minutes at $4^{\circ} \mathrm{C}$. Next, the biotinylation solution was removed and the reaction was quenched by the addition of HBSS supplemented with $10 \mathrm{mM}$ glycine, which was incubated at room temperature for 20 minutes with gentle shaking. Cells were then washed twice with $10 \mathrm{~mL}$ of HBSS for 5 minutes at room temperature. Cell viability was verified on control samples with a live/dead stain (Invitrogen). For affinity purification experiments, the cells from each flask were harvested into $5 \mathrm{~mL}$ of Guanidinium Lysis Buffer (GLB) (140 mM NaCl, $10 \mathrm{mM}$ $\mathrm{KCl}, 20 \mathrm{mM}$ NaPhosphate, $10 \mathrm{mM}$ EDTA, $2 \mathrm{mM}$ EGTA, $0.1 \%$ Tween-20, $6 \mathrm{M}$ Guanidinium $\mathrm{HCl}, \mathrm{pH}$ 7.2). For Western Blot experiments, cells were harvested into $4 \mathrm{ml}$ of RIPA buffer (Sigma, Saint Louis, MO) supplemented with protease inhibitor cocktail (Fermentas, Waltham, MA).

Insoluble debris in the whole cell lysate was pelleted by centrifugation for 10 minutes at 9,400 RCF at $4^{\circ} \mathrm{C}$. DNA and other soluble biopolymers were removed by syringe filtration through a $0.2 \mu \mathrm{m}$ filter. From each sample, $3 \mathrm{ml}$ of lysate in GLB buffer was transferred to a clean tube and $100 \mu \mathrm{l}$ of Streptavidin-coated C1 Dynabeads (Life Technologies, Grand Island, NY) was added to each tube. Samples were incubated at $23^{\circ} \mathrm{C}$ with agitation for $1 \mathrm{hr}$. Samples were washed twice for 10 minutes each with $1 \mathrm{ml}$ of GLB and were then washed twice for 30 minutes each with WB-PBS (140 mM NaCl, 20 mM NaPhosphate, 0.05\% Tween-20, $\mathrm{pH}$ 7.2). After washing, the supernatant was removed and $20 \mu \mathrm{l}$ of $\mathrm{RB}$ solution (1\% SDS in de-ionized $\mathrm{H}_{2} 0$ ) was added and samples were incubated at $50^{\circ} \mathrm{C}$ for 10 minutes. After this initial incubation, $30 \mu \mathrm{l}$ of Laemmli buffer with 5\% BME (Bio-Rad) was added to each sample. Subsequently, samples were incubated at $50^{\circ} \mathrm{C}$ for 5 minutes and $70^{\circ} \mathrm{C}$ for 10 minutes. The Dynabeads were then pelleted by centrifugation and $40 \mu \mathrm{l}$ of supernatant from each sample was used for SDS-PAGE. The capture and washing of the Dynabeads was performed with a magnetic stand (Life Technologies, Grand Island, NY).

To remove impurities, samples were run approximately $1 \mathrm{~cm}$ into a 12\% SDS-PAGE gel (Bio-Rad, Hercules, CA). The proteins were then excised and digested in-gel using the ProteaseMAX/Trypsin gold system, following manufacturer protocols (Promega, Madison, WI). The resulting solution of eluted peptides was dehydrated to dryness by vacuum centrifugation at $23^{\circ} \mathrm{C}$.

Proteomic analyses were performed in the Oregon State University MS facility and core. The LTQ-FT mass spectrometer was operated using data-dependent MS/ MS acquisition with a MS precursor ion scan, performed in the ICR cell, from $350-2000 \mathrm{~m} / \mathrm{z}$ with the resolving power set to 100,000 at $\mathrm{m} / \mathrm{z} 400$, and MS/MS scans performed by the linear ion trap on the five most abundant doubly or triply charged precursor ions detected in the MS scan. A binary solvent system consisting of 
solvent A, $2 \%$ aqueous acetonitrile with $0.1 \%$ formic acid, and solvent $\mathrm{B}$, acetonitrile with $0.1 \%$ formic acid was used for the analyses. Tryptic peptides were loaded onto a peptide trapping column (Cap Trap, Michrom) and separated using a C18 column (Agilent Zorbax 300SB-C18, $250 \times 0.3 \mathrm{~mm}, 5 \mu \mathrm{m})$. Peptides were trapped and washed with $3 \%$ solvent $B$ for 3 min at a flow rate of $5 \mu \mathrm{L} / \mathrm{min}$. Peptide separation was achieved using a linear gradient from $10 \% \mathrm{~B}$ to $30 \% \mathrm{~B}$ at a flow rate of $4 \mu \mathrm{L} / \mathrm{min}$ over 102 minutes. LC-MS/MS analysis was conducted on a Thermo LTQ-FT MS instrument coupled to a Waters nanoAcquity UPLC system.

Thermo RAW data files were processed with Proteome Discoverer v1.3.0. For database searching Mascot (v2.3) was used to search against the canine protein database downloaded from NCBI website (http://www.ncbi.nlm. nih.gov/). The following parameters were used for database searching: the digestion enzyme was set to Trypsin/P and two missed cleavage sites were allowed. The precursor ion mass tolerance was set to $10 \mathrm{ppm}$, while fragment ion tolerance of 0.8 Da was used. Dynamic modifications that were considered: carbamidomethyl $(+57.02 \mathrm{Da})$ for cysteine and oxidation (+15.99 Da) for methionine, phosphorylation (+97.98 Da) of serine, threonine and tyrosine and biotinylation of lysine. Automatic target decoy search with $1 \%$ FRD was implemented into the Mascot search parameters. Scaffold_3.3.1 (Proteome Software, Portland, OR) was used for search data compilation and data evaluation with embedded X!Tandem database searching algorithm. For protein validation, peptide identification probability was set to $95 \%$ and protein identification probability was set to $99 \%$, and proteins with two or more peptide matches were selected as true identifications [37].

\section{qRT-PCR}

Cells were grown to $\sim 80 \%$ confluency on $10 \mathrm{~cm}^{2}$ tissue culture plates, washed once with PBS and harvested into $1 \mathrm{~mL}$ of TRIzol reagent (Life Technologies, Grand Island, NY, Grand Island, NY). RNA was isolated by the manufacturer's guidelines and the resulting RNA was quantified with the NanoDrop ND-1000 instrument. For cDNA synthesis 1000 ng of total RNA was reverse transcribed using Double Primed RNA to cDNA EcoDry ${ }^{\text {TM }}$ Premix (Clontech, Mountain View, CA). All qRT-PCR assays were run in a total reaction volume of $10 \mu \mathrm{L}$ comprised of $5 \mu \mathrm{L}$ Power Sybr PCR Master Mix (Applied Biosystems, Carlsbad, CA), $500 \mathrm{nM}$ of both forward and reverse primers and $1 \mu \mathrm{L}$ of cDNA template. Real time PCR was performed in MicroAmp optical 96-well reaction plates using StepOnePlus Real-Time PCR Systems (Applied Biosystems, Carlsbad, CA). The cycle threshold (CT) for each gene of interest was normalized to GAPDH. The resulting delta-CT value was used to determine the relative quantification (RQ) of each gene using $2^{\wedge}$ (-delta CT). All RQ values were then divided by the $R Q$ value of the highest expressing cell line and multiplied by 100 to determine relative differences in expression levels between the cell lines. All qRT-PCR primers were designed across exons in order to prevent background signal from any contaminating genomic DNA (Additional file 5: Table S5).

\section{Western blot}

Insoluble debris in the cell lysate was pelleted by centrifugation for 10 minutes at $10,000 \mathrm{RPM}$ at $4^{\circ} \mathrm{C}$. The supernatant was then transferred to a new tube. Protein concentrations were assessed by BCA assay (Thermo Scientific, Rockford, IL). Approximately $5 \mu \mathrm{g}$ of total protein was run in each lane. Proteins were denatured in Laemmli buffer with 5\% BME (Bio-Rad, Hercules, CA) and separated by SDS-PAGE. Proteins were transferred to nitrocellulose films using the iBlot system (Life Technologies, Grand Island, NY). Primary antibodies targeting Thrombospondin-1 (sc-12312), CD44 (sc18849), CYR61 (sc-8560), Notch 2 (sc-5545) and Plexin B2 (sc-34504) and GAPDH (sc-166574) (Santa Cruz Biotechnology, Santa Cruz, CA) were used at a dilution of 1:200 in PBS supplemented with $0.05 \%$ Tween-20 and $2 \%$ BSA. IRdye secondary antibodies (LI-COR, Lincoln, $\mathrm{NE}$ ) were used at a dilution of $1: 10,000$ in PBS supplemented with $0.05 \%$ Tween-20 and $2 \%$ BSA. Western blots were visualized and recorded on the LI-COR scanning system (LI-COR, Lincoln, NE). To visualize biotinylated proteins, streptavidin IRDye680 (LI-COR, Lincoln, NE) was used for Western blot at a concentration of 1:10,000. To visualize total protein, samples were separated by SDS-PAGE and visualized by silver stain (Bio-Rad, Hercules, CA).

\section{Immunocytochemistry}

Cells were cultured to approximately $80 \%$ confluency in a 96-well plate. Cells were incubated with primary antibodies (Rat IgG control (sc-2026), anti-CD44 (sc-18849), goat IgG control (sc-2028), anti-Thrombospondin-1 (sc12312), CYR61 (sc-8560), anti-PlexinB2 (sc-34504), rabbit IgG control (sc-2027) and anti-Notch2 (sc- sc-5545) at a dilution of $1: 200$ in complete RPMI, at $4^{\circ} \mathrm{C}$ for $30 \mathrm{~min}$ (Santa Cruz Biotechnology). Cells were washed then incubated with secondary antibody, anti-rat-IgG-IRdye 800 (926-32219), anti-goat-IgG-IRdye800 (926-32214) or antirabbit-IgG-IRdye800 (926-32211) a dilution of 1:1000 in complete RPMI, at $4^{\circ} \mathrm{C}$ for $30 \mathrm{~min}$ (LICOR). Cells were then washed extensively, the media replaced with $50 \mu \mathrm{l}$ HBSS per well, and imaged on a LICOR scanner (LICOR). Hoescht 33342 (Sigma) was then added at a concentration of $2.5 \mu \mathrm{g} / \mathrm{ml}$ and incubated for $5 \mathrm{~min}$ at room temp. Cells were then washed and the fluorescent signal from the Hoeschst stain was read on a fluorescent plate reader. The 
fluorescent signal from the secondary antibodies was then normalized against the signal for Hoeschst, to account for differences in the number of cells (Additional file 4). Each condition had four technical replicates, which were used to establish the error bars (standard deviation).

\section{Availability of supporting data}

The data sets supporting the results of this article are included within the article and its additional files.

\section{Additional files}

Additional file 1: Table S1. Mass spectrometry-identified cell surfaceexposed proteins. Merged list of total cell surface-exposed protein biotinylation/streptavidin affinity purification mass spectrometry results for cultured normal canine osteoblasts ( $\mathrm{CnOb}$ ) and two validated canine osteosarcoma cell lines (POS and HMPOS).

Additional file 2: Table S2. Biological replicates of mass spectrometryidentified cell surface-exposed proteins. Complete list, with each biological replicate, of identified cell surface-exposed protein biotinylation/streptavidin affinity purification mass spectrometry results for cultured normal canine osteoblasts (CnOb) and two validated canine osteosarcoma cell lines (POS and HMPOS).

Additional file 3: Table S3. Biological replicates of quantitative real-time PCR data. Complete data, with each biological replicate, of quantitative real-time PCR results of cultured normal canine osteoblasts (CnOb) and two validated canine osteosarcoma cell lines (POS and HMPOS).

Additional file 4: Primers used for quantitative real-time PCR of cultured normal canine osteoblasts ( $\mathrm{CnOb}$ ) and two validated canine osteosarcoma cell lines (POS and HMPOS).

Additional file 5: Table S5. Immunocytochemistry data sets. Complete data, with all replicates and all tested antibodies, of immunocytochemistry (ICC) results for normal canine osteoblasts (CnOb) and two validated canine osteosarcoma cell lines (POS and HMPOS).

\section{Competing interests}

The author(s) declare that they have no competing interests.

\section{Authors' contributions}

All authors (MM, IHM, MJM, CPG, BS, SB, SIW) participated in conceptual study design, interpretation of the data, provided revisions to the manuscript, and read and approved the final manuscript. Additional individual author contributions are as follows: CPG and IHM performed the cell cultures; IHM and MJM performed the biotinylation and streptavidin purification; SIW performed the mass spectrometry analysis; MM, IHM, and MJM primarily authored the manuscript text. All authors read and approved the final manuscript.

\section{Acknowledgements}

The authors would like to acknowledge Prof. Claudia Maier for her critical advice on the project. This project was supported in part by funding from the Oregon State University College of Veterinary Medicine. The authors acknowledge the Biomolecular Mass Spectrometry Core of the Environmental Health Sciences Center at Oregon State University (NIH/NIEHS P30ES000210). The content is solely the responsibility of the authors and does not necessarily represent the official views of NIEHS or NIH.

\section{Author details}

'Department of Clinical Sciences, College of Veterinary Medicine, Oregon State University, Corvallis, OR 97331, USA. ${ }^{2}$ EACRI, Providence Portland Medical Center, Portland, OR 97213, USA. ${ }^{3}$ Department of Chemistry, Oregon State University, Corvallis, OR 97331, USA. ${ }^{4}$ Present address: The Animal Cancer Center, College of Veterinary Medicine and Biomedical Sciences, Colorado State University, Fort Collins, CO 80523, USA.
Received: 18 February 2013 Accepted: 5 June 2013

Published: 13 June 2013

\section{References}

1. Dernell WS, Ehrhart NP, Straw RC, Vail DM: Tumors of the Skeletal System. In, Withrow \& MacEwen's Small Animal Clinical Oncology, Volume 1. 4th edition Edited by Withrow SJ, Vail DM. St. Louis, MO: Saunders Elsevier; 2007:540-582.

2. Liptak JM, Dernell WS, Ehrhart N, Withrow SJ: Canine appendicular osteosarcoma: diagnosis and palliative treatment. Compend Contin Educ Vet 2004, 26:172-182.

3. Liptak JM, Dernell WS, Ehrhart N, Withrow SJ, Seguin B, Walsh PJ, Kuntz CA: Canine appendicular osteosarcoma: curative-intent treatment. Compend Contin Educ Vet 2004, 26:186-196.

4. Saam DE, Liptak JM, Stalker MJ, Chun R: Predictors of outcome in dogs treated with adjuvant carboplatin for appendicular osteosarcoma: 65 cases (1996-2006). J Am Vet Med Assoc 2011, 238(2):195-206.

5. Leth-Larsen R, Lund RR, Ditzel HJ: Plasma membrane proteomics and its application in clinical cancer biomarker discovery. Mol Cell Proteomics MCP 2010, 9(7):1369-1382.

6. Sprenger RR, Jensen ON: Proteomics and the dynamic plasma membrane: Quo Vadis? Proteomics 2010, 10(22):3997-4011.

7. Shoeneman JK, Ehrhart EJ 3rd, Eickhoff JC, Charles JB, Powers BE, Thamm $\mathrm{DH}$ : Expression and function of survivin in canine osteosarcoma. Canc Res 2012, 72(1):249-259.

8. Schmit JM, Pondenis HC, Barger AM, Borst LB, Garrett LD, Wypij JM, Neumann ZL, Fan TM: Cathepsin K expression and activity in canine osteosarcoma. J Vet Intern Med 2012, 26(1):126-134.

9. McCleese JK, Bear MD, Kulp SK, Mazcko C, Khanna C, London CA: Met interacts with EGFR and Ron in canine osteosarcoma. Vet Comp Oncol 2013, 11(2):123-139.

10. Ferracini $R$, Angelini $P$, Cagliero E, Linari A, Martano M, Wunder J, Buracco P: MET oncogene aberrant expression in canine osteosarcoma. J Orthop Res 2000, 18(2):253-256.

11. Fieten H, Spee B, ljzer J, Kik MJ, Penning LC, Kirpensteijn J: Expression of hepatocyte growth factor and the proto-oncogenic receptor c-Met in canine osteosarcoma. Vet Pathol 2009, 46(5):869-877.

12. Kirpensteijn J, Kik M, Teske E, Rutteman GR: TP53 gene mutations in canine osteosarcoma. Vet Surg 2008, 37(5):454-460.

13. Loukopoulos P, Thornton JR, Robinson WF: Clinical and pathologic relevance of p53 index in canine osseous tumors. Vet Pathol 2003, 40(3):237-248

14. Flint AF, U'Ren L, Legare ME, Withrow SJ, Dernell W, Hanneman WH: Overexpression of the erbB-2 proto-oncogene in canine osteosarcoma cell lines and tumors. Vet Pathol 2004, 41(3):291-296.

15. MacEwen EG, Pastor J, Kutzke J, Tsan R, Kurzman ID, Thamm DH, Wilson M Radinsky R: IGF-1 receptor contributes to the malignant phenotype in human and canine osteosarcoma. J Cell Biochem 2004, 92(1):77-91.

16. Lana SE, Ogilvie GK, Hansen RA, Powers BE, Dernell WS, Withrow SJ: Identification of matrix metalloproteinases in canine neoplastic tissue. Am J Vet Res 2000, 61(2):111-114.

17. Loukopoulos P, Mungall BA, Straw RC, Thornton JR, Robinson WF: Matrix metalloproteinase-2 and -9 involvement in canine tumors. Vet Pathol 2003, 40(4):382-394.

18. Khanna C, Wan X, Bose S, Cassaday R, Olomu O, Mendoza A, Yeung C Gorlick R, Hewitt SM, Helman L: The membrane-cytoskeleton linker ezrin is necessary for osteosarcoma metastasis. Nat Med 2004, 10(2):182-186.

19. Josic D, Clifton JG: Mammalian plasma membrane proteomics. Proteomics 2007, 7(16):3010-3029.

20. Folio C, Mora MI, Zalacain M, Corrales FJ, Segura V, Sierrasesumaga L, Toledo G, San-Julian M, Patino-Garcia A: Proteomic analysis of chemonaive pediatric osteosarcomas and corresponding normal bone reveals multiple altered molecular targets. J Proteome Res 2009, 8(8):3882-3888

21. Guo QC, Shen JN, Jin S, Wang J, Huang G, Zhang L, Huang G, Yin JQ, Zou CY, Li MT: Comparative proteomic analysis of human osteosarcoma and SV40-immortalized normal osteoblastic cell lines. Acta Pharmacol Sin 2007, 28(6):850-858.

22. Hua $Y$, Jia $X$, Sun $M$, Zheng $L$, Yin $L$, Zhang $L$, Cai Z: Plasma membrane proteomic analysis of human osteosarcoma and osteoblastic cells: revealing NDRG1 as a marker for osteosarcoma. Tumour Biol I Int Soc Oncodevelopmental Biol Med 2011, 32(5):1013-1021. 
23. Zhang Z, Zhang L, Hua Y, Jia X, Li J, Hu S, Peng X, Yang P, Sun M, Ma F, et al: Comparative proteomic analysis of plasma membrane proteins between human osteosarcoma and normal osteoblastic cell lines. BMC Canc 2010, 10:206.

24. Peirce MJ, Wait R, Begum S, Saklatvala J, Cope AP: Expression profiling of lymphocyte plasma membrane proteins. Mol Cell Proteomics MCP 2004, 3(1):56-65.

25. Hua YQ, Jia XF, Sun MX, Zheng LP, Yin L, Zhang LJ, Cai ZD: Plasma membrane proteomic analysis of human osteosarcoma and osteoblastic cells: revealing NDRG1 as a marker for osteosarcoma. Tumor Biol 2011, 32(5):1013-1021.

26. Zhang $Z Y$, Zhang $L$, Hua YQ, Jia XF, Li JA, Hu S, Peng X, Yang PY, Sun MX, $\mathrm{Ma} F$, et al: Comparative proteomic analysis of plasma membrane proteins between human osteosarcoma and normal osteoblastic cell lines. BMC Canc 2010, 10:206.

27. Allen M, Louise Jones J: Jekyll and Hyde: the role of the microenvironment on the progression of cancer. J Pathol 2011, 223(2):162-176.

28. Deng S, Wang J, Hou L, Li J, Chen G, Jing B, Zhang X, Yang Z: Annexin A1, A2, A4 and A5 play important roles in breast cancer, pancreatic cancer and laryngeal carcinoma, alone and/or synergistically. Oncol Lett 2013, 5(1):107-112.

29. Haque I, De A, Majumder M, Mehta S, McGregor D, Banerjee SK, Van Veldhuizen P, Banerjee S: The matricellular protein CCN1/Cyr61 is a critical regulator of Sonic Hedgehog in pancreatic carcinogenesis. J Biol Chem 2012, 287(46):38569-38579.

30. Pirazzoli V, Sarra Ferraris GM, Sidenius N: Direct evidence of the importance of vitronectin and its interaction with the urokinase receptor in tumor growth. Blood 2013, :

31. Tan HT, Lee YH, Chung MC: Cancer proteomics. Mass Spectrom Rev 2012, 31(5):583-605.

32. Blacking TM, Waterfall M, Argyle DJ: CD44 is associated with proliferation, rather than a specific cancer stem cell population, in cultured canine cancer cells. Vet Immunol Immunopathol 2011, 141(1-2):46-57.

33. Mi W, Jia W, Zheng Z, Wang J, Cai Y, Ying W, Qian X: Surface glycoproteomic analysis of hepatocellular carcinoma cells by affinity enrichment and mass spectrometric identification. Glycoconj J 2012, 29(5-6):411-424.

34. Chen $R$, Jiang $X$, Sun $D$, Han $G$, Wang F, Ye M, Wang L, Zou H: Glycoproteomics analysis of human liver tissue by combination of multiple enzyme digestion and hydrazide chemistry. J Proteome Res 2009, 8(2):651-661.

35. Gilmore JM, Kettenbach AN, Gerber SA: Increasing phosphoproteomic coverage through sequential digestion by complementary proteases. Anal Bioanal Chem 2012, 402(2):711-720.

36. Barroga EF, Kadosawa T, Okumura M, Fujinaga T: Establishment and characterization of the growth and pulmonary metastasis of a highly lung metastasizing cell line from canine osteosarcoma in nude mice. J Veterinary Med Sci Jpn Soc Veterinary Sci 1999, 61(4):361-367.

37. Wickramasekara S, Neilson J, Patel N, Breci L, Hilderbrand A, Maier RM, Wysocki V: Proteomics analyses of the opportunistic pathogen Burkholderia vietnamiensis using protein fractionations and mass spectrometry. J Biomed Biotechnol 2011, 2011:701928.

\section{doi:10.1186/1746-6148-9-116}

Cite this article as: Milovancev et al: Comparative analysis of the surface exposed proteome of two canine osteosarcoma cell lines and normal canine osteoblasts. BMC Veterinary Research 2013 9:116.

\section{Submit your next manuscript to BioMed Central and take full advantage of:}

- Convenient online submission

- Thorough peer review

- No space constraints or color figure charges

- Immediate publication on acceptance

- Inclusion in PubMed, CAS, Scopus and Google Scholar

- Research which is freely available for redistribution

Submit your manuscript at www.biomedcentral.com/submit 